\title{
ON A CON JECTURE CONCERNING PLANAR COVERINGS OF SURFACES
}

\author{
BY BERNARD MASKIT ${ }^{1}$
}

Communicated by Lipman Bers, January 29, 1963

C. D. Papakyriakopoulos [1] recently proposed two conjectures in conjunction with his work on the Poincare conjecture. We present here three counter examples to the second of these. He has subsequently modified his conjectures [2] as suggested by these examples. The conjecture to be contradicted is the following.

Let $S$ be a closed orientable surface of genus $g \geqq 2$. Let $A_{1}, B_{1}, \cdots$, $A_{a}, B_{0}$ be a fundamental system of $S$ based at o [1, p. 360]. Let $a_{1}, b_{1}, \cdots, a_{g}, b_{g}$ be the elements of $\pi_{1}(S, o)$ corresponding to $A_{1}, B_{1}, \cdots, A_{g}, B_{g}$ respectively; then

$$
\pi_{1}(S, o) \simeq F=\left(a_{1}, b_{1}, \cdots, a_{\theta}, b_{\theta}: \prod_{i=1}^{o}\left[a_{i}, b_{i}\right] .\right)
$$

Let $\phi$ be the free group freely generated by $a_{1}, b_{1}, \cdots, a_{\theta}, b_{0}$. Let $\tau_{j}$ be a word in the $a$ 's and b's representing an element of $[\phi, \phi], j=1, \cdots, g$. Then for some subset $(m, \cdots, n)$ of $(1, \cdots, g)$ the regular covering surface $\tilde{S}$ of $S$, corresponding to $\left\langle\left[a_{m}, b_{m} \tau_{m}\right], \cdots,\left[a_{n}, b_{n} \tau_{n}\right]\right\rangle$ in $F$ $[1$, p. 361 , footnote 5$]$, is planar.

The three examples are differentiated by the following properties.

A. The elements $b_{j} \tau_{j}$ in $F, j=1, \cdots, g$, can be represented by simple loops on $S[1$, p. 365].

$\mathrm{B}$. The words $b_{j} \tau_{j}$ in the $a$ 's and $b$ 's are cyclically reduced.

In all three examples we take $S$ of genus 2 , with the basis $A_{1}, B_{1}$, $A_{2}, B_{2}$ as shown in Figure 1. For the first example we take $\tau_{1}=\left[b_{1}^{-1}, b_{2}\right]$, $\tau_{2}=\left[b_{2}^{-1}, b_{1}\right]$; this satisfies $\mathrm{A}$ but not $\mathrm{B}$. In the second example $\tau_{1}=\left[b_{2}^{-1}, a_{1}^{-1}\right], \tau_{2}=\left[b_{1}^{-1}, a_{2}^{-1}\right]$; this satisfies B but not A. In the third example $\tau_{1}=\left[b_{2}, a_{2}\right], \tau_{2}=\left[b_{1}, a_{1}\right]$; this satisfies both $\mathrm{A}$ and $\mathrm{B}$.

We present here a proof only for the third counter example. The proofs for the first two are essentially the same except that, for these, one does not need the explicit construction of a certain group, and in the second example there are 19 cases to consider, while there are 7 cases in both the first and third.

We now assume that $\widetilde{S}_{1}$, the regular covering surface of $S$ corresponding to $\left\langle\left[a_{1}, b_{1} \tau_{1}\right]\right\rangle\left(\tau_{1}=\left[b_{2}, a_{2}\right]\right)$, is planar. Let $C_{1}$ be a loop on

1 This research was supported by the Office of Naval Research under Contract No. NONR-285(46) and by the National Science Foundation. The author is currently a National Science Foundation Graduate Fellow. 


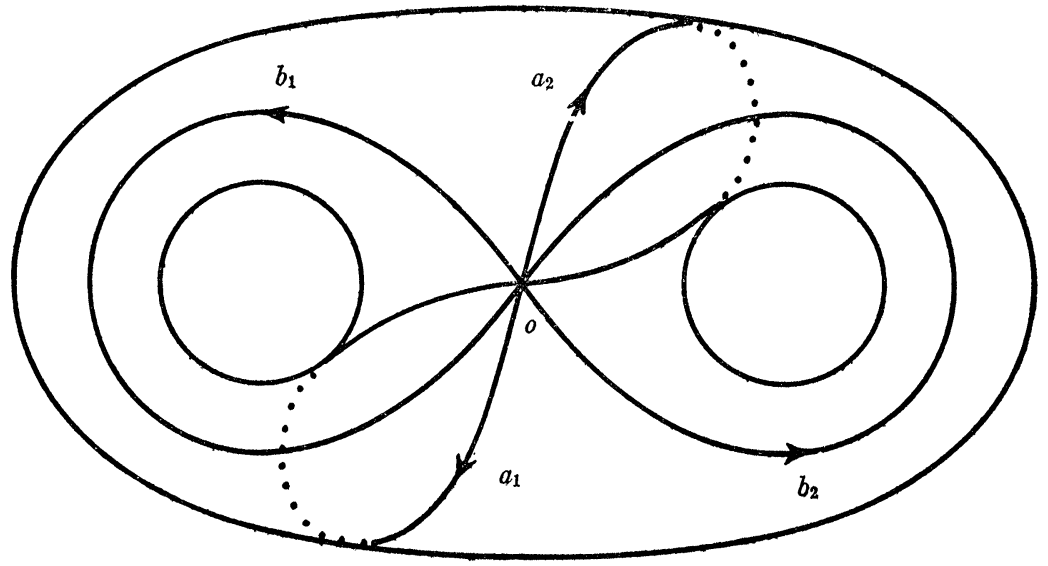

Figure 1

$S$ representing $\left[a_{1}, b_{1} \tau_{1}\right]$ as shown in Figure 2, and let $C_{2}$ be a directed curve "parallel" to $C_{1}$. Let $o$ be the point of intersection of $C_{1}$ and $C_{2}$ marked in Figure 2. We now lift $C_{1}$ and $C_{2}$ to $\widetilde{C}_{1}$ and $\widetilde{C}_{2}$ respectively, starting at a point $\tilde{o}$ over $o$. Since $\widetilde{S}_{1}$ is planar, $\widetilde{C}_{1}$ and $\widetilde{C}_{2}$ must have a second point of intersection $\widetilde{P}$ which projects to a point of intersection $P$, of $C_{1}$ and $C_{2}$. If we orient $S$ and lift the orientation to $\widetilde{S}_{1}$, then we can choose $\widetilde{P}$ so that the sense of intersection at $\widetilde{P}$ is the reverse of that at $\delta$, and by projection, the senses of intersection at $P$ and $o$ are reversed. Therefore $P$ must be one of the points marked $1, \cdots, 7$ in Figure 2. Furthermore, if we follow $C_{1}$ from o to $P$ and $C_{2}$ from $P$ to $o$, then the element of $F$ corresponding to this loop lies in the defining subgroup for $\tilde{S}_{1}$.

We now have seven cases to consider. If, for example, $P$ is the point marked 1 , then the element of $F$ obtained by the above construction is $b_{1}^{-1} a_{1} b_{1}$. But $b_{1}^{-1} a_{1} b_{1}$ cannot be in $\left\langle\left[a_{1}, b_{1} \tau_{1}\right]\right\rangle$, since the element of $\phi$ corresponding to the word $b_{1}^{-1} a_{1} b_{1}$ does not belong to $[\phi, \phi]$. The same reasoning shows that $P$ cannot be any of the points marked $2, \cdots, 6$. Hence $P$ must be the point marked 7 . Therefore the above construction gives us that $\tau_{1} \in\left\langle\left[a_{1}, b_{1} \tau_{1}\right]\right\rangle$, i.e. $\tau_{1}$ belongs to the smallest normal subgroup of $F$ containing $\left[a_{1}, b_{1} \tau_{1}\right]$.

Nothing in the above is changed if we replace $\widetilde{S}_{1}$ by $\tilde{S}_{2}$, the regular covering surface corresponding to $\left\langle\left[a_{1}, b_{1} \tau_{1}\right],\left[a_{2}, b_{2} \tau_{2}\right]\right\rangle$. Also if we look at Figure 2 upside down, the above construction shows that if $\widetilde{S}_{3}$, corresponding to $\left\langle\left[a_{2}, b_{2} \tau_{2}\right]\right\rangle$, is planar, then $\tau_{2}=\tau_{1}^{-1}$ is in $\left\langle\left[a_{2}, b_{2} \tau_{2}\right]\right\rangle$.

The relation, $\tau_{1} \in\left\langle\left[a_{1}, b_{1} \tau_{1}\right]\right\rangle$, implies that $\tau_{1}$ must be the identity in the group 


$$
G=\left(a_{1}, b_{1}, a_{2}, b_{2}:\left[a_{1}, b_{1}\right]\left[a_{2}, b_{2}\right],\left[a_{1}, b_{1} \tau_{1}\right],\left[a_{2}, b_{2} \tau_{2}\right]\right)
$$

where $\tau_{1}=\left[b_{2}, a_{2}\right]$ and $\tau_{2}=\left[b_{1}, a_{1}\right]$. Let us now consider a group $\Gamma$ of $2 \times 2$ matrices on generators

$$
\alpha_{1}=\left(\begin{array}{ll}
1 & 1 \\
0 & 1
\end{array}\right), \quad \beta_{1}=\left(\begin{array}{rr}
1 & 0 \\
-1 & 1
\end{array}\right), \quad \alpha_{2}=\left(\begin{array}{rr}
0 & 1 \\
-1 & -2
\end{array}\right), \quad \beta_{2}=\left(\begin{array}{rr}
-1 & 0 \\
-1 & -1
\end{array}\right)
$$

and the map $G \rightarrow \Gamma$ defined by

$$
a_{1} \rightarrow \alpha_{1}, \quad b_{1} \rightarrow \beta_{1}, \quad a_{2} \rightarrow \alpha_{2}, \quad b_{2} \rightarrow \beta_{2} .
$$

This is a homomorphism, since

$$
\left[\alpha_{1}, \beta_{1}\right]\left[\alpha_{2}, \beta_{2}\right]=1, \quad \alpha_{1}=\beta_{1}\left[\beta_{2}, \alpha_{2}\right], \quad \alpha_{2}=\beta_{2}\left[\beta_{1}, \alpha_{1}\right]
$$

as one can easily see. However, $\tau_{1} \rightarrow\left[\beta_{2}, \alpha_{2}\right] \neq 1$. We have arrived at a contradiction.

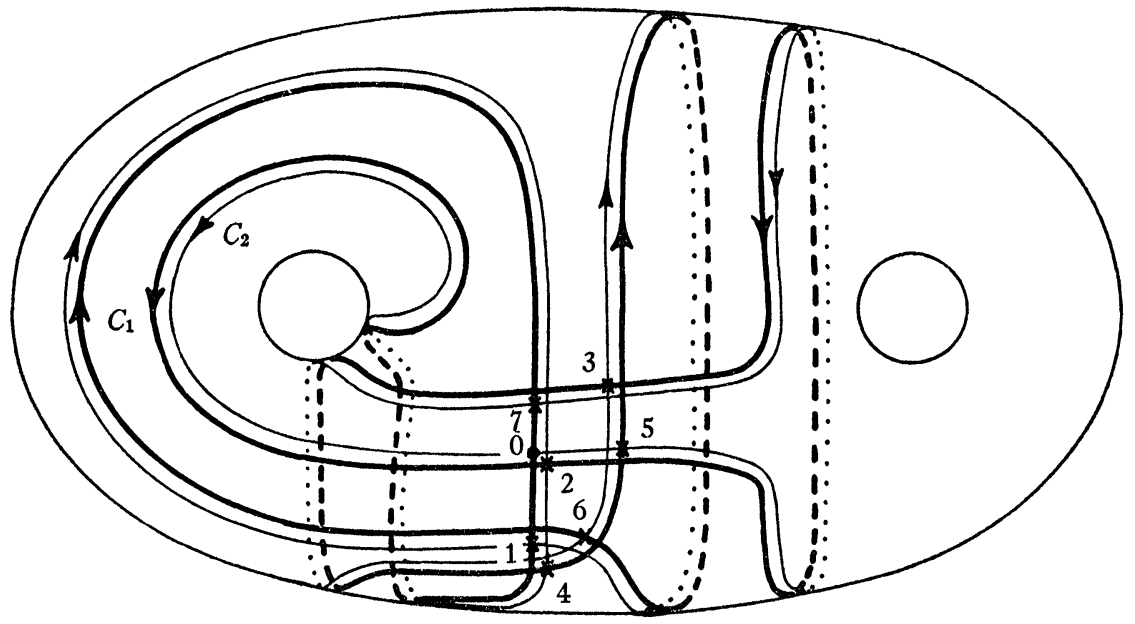

FIGURE 2

The assumption that $\tilde{S}_{1}$ is planar leads to a contradiction. Hence $\tilde{S}_{1}$ is not planar.

The author wishes to thank Professor L. Bers and Dr. Papakyriakopoulos for many informative conversations.

\section{REFERENCES}

1. C. D. Papakyriakopoulos, $A$ reduction of the Poincare conjecture to other conjectures, Bull. Amer. Math. Soc. 68 (1962), 360-366.

2. $-A$ reduction of the Poincare conjecture to other conjectures. II, Bull. Amer. Math. Soc. 69 (1963), 399-401.

NeW YoRk UNIVERSITY 